\title{
Correction: Identification and replication of RNA- Seq gene network modules associated with depression severity
}

Trang T. Le, Jonathan Savitz, Hideo Suzuki, Masaya Misaki, T. Kent Teague, Bill C. White (D, Julie H. Marino, Graham Wiley, Patrick M. Gaffney, Wayne C. Drevets, Brett A. McKinney and Jerzy Bodurka

\section{Correction to: Translational Psychiatry https://doi.org/10.1038/s41398-018-0234-3 published online 5 September 2018}

This Article has been corrected to clear up scientific details (see the authors' summary below).

"Our analysis used stranded RNA-Seq preprocessing where the forward direction was used for the second fast sequence files. This stranded preprocessing enriches for antisense non-coding RNA, sometimes called Natural Antisense Transcripts (NATs). These NATs are labeled with AS1 (for antisense) appended to their gene symbols, and they are known to recruit epigenetic machinery and other mechanisms to regulate coding RNA (mRNA/ genes). In addition to NATs, stranded preprocessing enriches for protein coding genes that can be transcribed in the antisense direction, which occurs for a significant proportion of mammalian genes (i.e., protein coding). Thus, the replicated module (M5) contains genes that are enriched for antisense expression of protein coding genes and expression of NATs that regulate partner coding genes through an antisense mechanism. We include the RNA-Seq data preprocessed for both antisense RNA and sense RNA gene expression in the github repository (https://github.com/insilico/DepressionGeneModules)."

Published online: 12 August 2020 